Pacific Journal of Mathematics

COLLECTIONS AND SEQUENCES OF CONTINUA IN THE 


\title{
COLLECTIONS AND SEQUENCES OF CONTINUA IN THE PLANE. II
}

\author{
C. E. Burgess
}

1. Introduction. This paper includes a study of some convergence properties of sequences of mutually exclusive continua in $E^{2}$, and these results are used to obtain some restrictions on the types of continua in an uncountable collection of mutually exclusive continua in $E^{2}$. The concept of width of a tree-like continuum is introduced, and it is shown that $E^{2}$ does not contain uncountably many mutually exclusive tree-like continua with positive widths. This gives a generalization of $\mathrm{R}$. L. Moore's result that $E^{2}$ does not contain uncountably many mutually exclusive triodic continua [13]. The author has presented some related results in [7].

Definitions of trees, chains, tree-like continua, and triods can be found in [8].

2. The width of a tree-like continuum. If $G$ is a tree, then a number $\mathscr{W}(G)$ is associated with $G$ as follows. For each chain $C$ in $G$ and each element $X$ of $G$, there is a distance ${ }^{1} \rho\left(X, C^{*}\right)$ from $X$ to $C^{*}$. Let,

$$
\mathscr{V}(G)=\min _{\sigma \text { in } G}\left[\max _{x \in G} \rho\left(X, C^{*}\right)\right],
$$

where each maximum is obtained with $C$ fixed. A number $w$ is called the width of a tree-like continuum $M$ if, for any cofinal sequence $G_{1}, G_{2}, G_{3}, \cdots$ of trees defining $M$, the sequence $\mathscr{W}\left(G_{1}\right), \mathscr{W}_{(}\left(G_{2}\right), \mathscr{W}\left(G_{3}\right), \cdots$ converges to $w$.

\section{THEOREM 1. Every tree-like continuum has a width.}

Proof. Suppose that some tree-like continuum $M$ does not have a width. Then there exist two nonnegative numbers $w_{1}$ and $w_{2}\left(w_{1}<w_{2}\right)$ and two cofinal sequences $G_{1}, G_{2}, G_{3}, \cdots$ and $H_{1}, H_{2}, H_{3}, \cdots$ of trees defining $M$ such that the sequence $\mathscr{W}\left(G_{1}\right), \mathscr{W}\left(G_{2}\right), \mathscr{W}\left(G_{3}\right), \cdots$ converges to $w_{1}$ and the sequence $\mathscr{W}\left(H_{1}\right), \mathscr{W}\left(H_{2}\right), \mathscr{W}\left(H_{3}\right), \cdots$ converges to $w_{2}$. Let $\varepsilon$ be a positive number such that

$$
3 \varepsilon<w_{2}-w_{1} .
$$

Received May 26, 1960. Presented to the American Mathematical Society, November 21, 1959, and August 30, 1960, under different titles. This work was supported by the National Science Foundation under G-5880.

1 The point set which is the sum of the elements of $C$ is denoted by $C^{*}$. 
Let $i$ be an integer such that the mesh of $G_{i}$ is less than $\varepsilon / 2$ and

$$
\left|\mathscr{Y}\left(G_{i}\right)-w_{1}\right|<\varepsilon \text {. }
$$

There exists an integer $j$ such that $H_{j}$ is a refinement of $G_{i}$ and

$$
\left|\mathscr{\mathscr { V }}\left(H_{j}\right)-w_{2}\right|<\varepsilon .
$$

Let $C$ be a chain in $G_{i}$ such that

$$
\mathscr{W}\left(G_{i}\right)=\max _{x \in G_{i}} \rho\left(X, C^{*}\right) .
$$

There is a chain $D$ in $H_{j}$ such that each link of $C$ contains a link of $D$. Let $Y$ be an element of $H_{j}$ such that

$$
\rho\left(Y, D^{*}\right)=\max _{x \in H_{j}} \rho\left(X, D^{*}\right),
$$

and let $Z$ be an element of $G_{i}$ that contains $Y$. Then

$$
\rho\left(Y, D^{*}\right) \leqq \rho\left(Z, C^{*}\right)+\varepsilon .
$$

It follows from (2) and (4) that

$$
\rho\left(Z, C^{*}\right)<w_{1}+\varepsilon .
$$

Now (6) and (7) imply that

$$
\rho\left(Y, D^{*}\right)<w_{1}+2 \varepsilon .
$$

Hence $D$ is a chain in $H_{j}$ such that

$$
\max _{x \in H_{j}} \rho\left(X, D^{*}\right)<w_{1}+2 \varepsilon,
$$

and since

$$
\mathscr{\mathscr { V }}\left(H_{j}\right) \leqq \max _{X \in H_{j}} \rho\left(X, D^{*}\right),
$$

it follows from (9) that

$$
\mathscr{W}\left(H_{j}\right)<w_{1}+2 \varepsilon .
$$

Now a combination of (3) and (11) gives

$$
w_{2}<w_{1}+3 \varepsilon,
$$

and this is contrary to (1).

CoRollary. Every linearly chainable continuum has width zero.

REMARK. There exists a tree-like continuum which has width zero and which is not linearly chainable. A continuum which is the sum of a 
simple triod $T$ and a ray spiralling around $T$ is such an example. Any tree-like continuum which is almost chainable [9] has width zero.

THEOREM 2. If the tree-like continuum $M$ has width zero, then every homeomorphic image of $M$ has width zero.

Proof. In order that a tree-like continuum $K$ should have width zero it is necessary and sufficient that, for every positive number $\varepsilon$, there should exist an $\varepsilon$-tree $G$ covering $K$ and a chain $C$ in $G$ such that every point of $K$ is within a distance $\varepsilon$ of some link of $C$. Hence, Theorem 2 follows from the fact that every homeomorphism of $M$ is uniformly continuous.

\section{THEOREM 3. Every tree-like triod has a positive width.}

Proof. Suppose that some tree-like triod $M$ has width zero. Then for each positive number $\varepsilon$, there exist an $\varepsilon$-tree $G$ covering $M$ and a chain $C$ in $G$ such that every point of $M$ is within a distance $\varepsilon$ of some link of $C$. A contradiction can be reached by using an argument similar to the proof of Theorem 6 of [9].

3. Convergent sequences of continua in $E^{2}$. A sequence of continua $M_{1}, M_{2}, M_{3}, \ldots$ is said to converge homeomorphically to a continuum $M$ if, for each positive number $\varepsilon$, there exists an integer $k$ such that, for $n>k$, there is a homeomorphism of $M_{n}$ onto $M$ that moves no point more than a distance $\varepsilon$.

THeOREM 4. If $M_{1}, M_{2}, M_{3}, \cdots$ is a sequence of mutually exclusive tree-like continua in $E^{2}$ converging to a continuum $M$ and, for each $i, w_{i}$ is the width of $M_{i}$, then the sequence $w_{1}, w_{2}, w_{3}, \cdots$ converges to zero.

The following lemma will be used in the proof of this theorem.

LEMMA. If $n$ is a positive integer, $H$ is a collection consisting of $n$ mutually exclusive closed disks in $E^{2}$, and $K$ is a collection consisting of $n^{3}$ mutually exclusive dendrons in $E^{2}$ such that each element of $K$ intersects every element of $H$, then some element of $K$ contains an arc which intersects every element of $H$.

Proof. The case where $n=1$ is trivial, so suppose that $n>1$. Each element of $K$ contains a dendron which is irreducible among the elements of $H$. Hence there exists a collection $K^{\prime}$ consisting of $n^{3}$ mutually exclusive dendrons such that each element of $K^{\prime}$ is irreducible among the elements of $H$ and is a subset of an element of $K$. Now 
since $n^{3}$ is greater than the product of $2 n$ and the number of pairs of elements of $H$, it follows from [7, Theorem 3] that there exist two elements $D_{1}$ and $D_{2}$ of $H$ and a collection $K^{\prime \prime}$ consisting of $2 n$ elements of $K^{\prime}$ such that each element of $K^{\prime \prime}$ intersects each element of $H$ and is a subset of $\mathrm{cl}\left[E^{2}-\left(D_{1}+D_{2}\right)\right]$. Now it follows from [6, Theorem 4] that some element of $K^{\prime \prime}$ contains an arc which intersects every element of $H$. Hence some element of $K$ contains such an arc.

Proof of Theorem 4. Suppose that the sequence $w_{1}, w_{2}, w_{3}, \cdots$ does not converge to zero, and for convenience suppose that there is a positive number $\delta$ such that each $w_{i}$ is greater than $\delta$. Let $\varepsilon$ be a positive number less than $\delta / 4$. There exists a finite set $B$ consisting of $n$ points of $M$ such that every point of $M$ is within a distance $\varepsilon$ of some point of $B$. There exist a collection $G$ of open disks of diameter less than $\varepsilon$ and a subcollection $G^{\prime}$ of $G$ such that

(1) $G$ is an essential covering of $M$,

(2) $G^{\prime}$ is an essential covering of $B$, and

(3) the closures of the elements of $G^{\prime}$ are mutually exclusive. Let $G^{\prime \prime}$ denote the collection of all closed disks which are the closures of the open disks of $G^{\prime}$. There exists an integer $k$ such that, for $i \geqq k$, $G$ is an essential covering of $M_{i}$. Now for each $i \quad\left(k \leqq i \leqq k+n^{3}\right)$, there exists a tree $G_{i}$ such that

(1) $G_{i}$ is an essential covering of $M_{i}$,

(2) each element of $G_{i}$ is an open disk,

(3) $G_{i}$ is a refinement of $G$,

(4) no element of $G_{i}$ intersects an element of $G_{j}$ for $j \neq i$,

(5) if $C_{i}$ is a linear chain in $G_{i}$, some element of $G_{i}$ is a distance greater than $\delta$ from $C_{i}^{*}$, and

(6) the nerve of $G_{i}$ can be realized by a dendron $K_{i}$ which is covered essentially by $G_{i}$ and which has a width greater than $\delta$.

It follows from the above lemma that for same integer $s\left(k \leqq \mathrm{~s} \leqq k+n^{3}\right)$, there is an arc $T_{s}$ in $K_{s}$ which intersects every element of $G^{\prime \prime}$. Requirement (6) implies that some point $p$ of $K_{s}$ is a distance greater than $\delta$ from $T_{s}$. Let $q$ be a point of $M_{s}$ such that $\rho(p, q)=\rho\left(p, M_{s}\right)$, let $r$ be a point of $M$ such that $\rho(q, r)=\rho(q, M)$, and let $u$ be a point of $B$ such that $\rho(r, u)=\rho(r, B)$. Now since $\rho\left(p, M_{s}\right)<\varepsilon, \rho(q, M)<\varepsilon, \rho(r, B)<\varepsilon$, and $\rho\left(u, T_{s}\right)<\varepsilon$, this leads to the contradiction that $\rho\left(p, T_{s}\right)<\delta$. Hence, the sequence $w_{1}, w_{2}, w_{3}, \cdots$ converges to zero.

TheOREM 5. If $M_{1}, M_{2}, M_{3}, \cdots$ is a sequence of mutually exclusive tree-like continua in $E^{2}$ converging homeomorphically to a continuum $M_{0}$, then the width of each $M_{i}$ is zero.

Proof. Let $\varepsilon$ be a positive number. It follows from Theorem 4 and 
the homeomorphic convergence of the sequence $M_{1}, M_{2}, M_{3}, \ldots$ that there exist a positive integer $n$, a tree $G_{n}$ covering $M_{n}$, and a homeomorphism $f$ of $M_{n}$ onto $M_{0}$ such that $\varepsilon / 3$ is greater than each of the width of $M_{n}$, the number $\mathscr{\mathscr { W }}\left(G_{n}\right)$, the mesh of $G_{n}$, and the distance any point of $M_{n}$ is moved under $f$. Let $C_{n}$ be a chain in $G_{n}$ such that

$$
\mathscr{\mathscr { V }}\left(G_{n}\right)=\max _{x \in G_{n}} \rho\left(X, C_{n}^{*}\right) \text {. }
$$

Now let $G$ denote the tree which is the collection of all images, under $f$, of elements of $G_{n}$, and let $C$ denote the chain in $G$ which consists of all images, under $f$, of elements of $C_{n}$. It follows that the mesh of $G$ is less than $\varepsilon$ and that for each element $Y$ in $G_{n}$,

$$
\rho\left(f(Y), C^{*}\right)<\rho\left(Y, C_{n}^{*}\right)+2 \varepsilon / 3 .
$$

A combination of (1) and (2) gives

$$
\rho\left(f(Y), C^{*}\right)<\mathscr{W}\left(G_{n}\right)+2 \varepsilon / 3 .
$$

Now since $\mathscr{W}\left(G_{n}\right)<\varepsilon / 3$, it follows from (3) that

$$
\rho\left(f(Y), C^{*}\right)<\varepsilon .
$$

Hence it has been shown that for each positive number $\varepsilon$, there is an $\varepsilon$-tree $G$ covering $M_{0}$ such that $\mathscr{W}^{-}(G)<\varepsilon$, and from this it follows that $M_{0}$ has width zero. That the width of each $M_{i}$ is zero follows from Theorem 2.

TheOREM 6. If $M_{1}, M_{2}, M_{3}, \cdots$ is a sequence of mutually exclusive continua in $E^{2}$ converging homeomorphically to a continuum $M_{0}$, then no $M_{i}$ has more than two complementary domains.

Proof. Suppose that $M_{0}$ has three complementary domains. Let $a, b$, and $c$ be three points in the complement of $M_{0}$ such that no two of them are in the same complementary domain of $M_{0}$, and let $\varepsilon$ be a positive number that is less than the distance from $M_{0}$ to $a+b+c$. There exists an integer $k$ such that, for $n>k$, there is a homeomorphism $f_{n}$ of $M_{n}$ onto $M_{0}$ that moves no point more than a distance $\varepsilon / 2$, and hence, for $n>k, M_{n}$ does not contain one of the points $a, b$, and $c$. Now let $h$ and $j$ be two integers greater than $k$. It follows from a theorem proved by Eilenberg [10, Theorem 5] that each of the continua $M_{h}$ and $M_{j}$ separates each two of the points $a, b$, and $c$ in $E^{2}$. On the other hand, $M_{h}$ and $M_{\jmath}$ are mutually exclusive so that $M_{h}$ would lie in some complementary domain of $M_{j}$, and hence some two of the points $a, b$, and $c$ would not be separated by $M_{h}$. From this contradiction, it follows that $M_{0}$ does not have more than two complementary domains. Consequently, no $M_{i}$ has more than two complementary domains. 
THEOREM 7. If $M_{1}, M_{2}, M_{3}, \cdots$ is a sequence of mutually exclusive continua in $E^{2}$ converging homeomorphically to a continuum $M_{0}$ that separates $E^{2}$, then each $M_{i}$ irreducibly separates $E^{2}$ into two components.

Proof. It follows from Theorem 6 that $M_{0}$ separates $E^{2}$ into two components, so suppose that some proper subcontinuum of $M_{0}$ separates $E^{2}$. Then some proper subcontinuum $K$ of $M_{0}$ would irreducibly separate $E^{2}$ into two components. Let $p$ be a point of $M_{0}-K$, let $q$ be a point that is separated in $E^{2}$ from $p$ by $K$, and let $\varepsilon$ be a positive number less than the distance from $K$ to $p+q$. Let $D$ be an open circular disk with center at $p$ and with radius $\varepsilon / 3$. There exist integers $h$ and $j$ such that the continua $M_{h}$ and $M_{j}$ are carried onto $M_{0}$ by homeomorphisms $f_{h}$ and $f_{j}$, respectively, that move no point more than a distance $\varepsilon / 3$. Let $K_{h}$ and $K_{j}$ denote the continua $f_{h}^{-1}(K)$ and $f_{j}^{-1}(K)$, respectively. Each of the continua $M_{h}$ and $M_{j}$ intersects $D$ and, by [10,

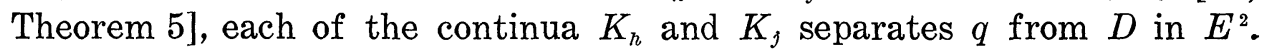
Since $K_{h}$ and $K_{j}$ are mutually exclusive, it follows that one of them, say $K_{h}$, separates the other, $K_{j}$, from $D$ in $E^{2}$. This involves the contradiction that $M_{j}$ intersects both $K_{j}$ and $D$ but does not intersect $K_{h}$. Hence, it follows that each $M_{i}$ irreducibly separates $E^{2}$ into two components.

THeORem 8. There does not exist in $E^{2}$ a sequence of mutually exclusive triods converging homeomorphically.

Proof. Let $M_{1}, M_{2}, M_{3}, \cdots$ be a sequence of mutually exclusive continua in $E^{2}$ converging homeomorphically to a continuum $M$. It is sufficient to show that $M$ is not a triod.

Case 1. The continuum $M$ separates $E^{2}$. By Theorem 7, $M$ irreducibly separates $E^{2}$ into two components so that no proper subcontinuum of $M$ separates $M[12]$. Hence $M$ is not a triod.

Case 2. The continuum $M$ does not separate $E^{2}$. Then $M$ is treelike as it contains no open subset of $E^{2}[2]$. By Theorem 5, $M$ has width zero, and hence it follows from Theorem 3 that $M$ is not a triod.

4. Uncountable collections of mutually exclusive continua in $E^{2}$. Roberts [14] has shown that every linearly chainable continuum has uncountable many mutually exclusive homeomorphic images in $E^{2}$. However, this is not the case for tree-like continua with width zero as the continuum described in the remark in $\S 1$ has width zero and contains. a simple triod. Anderson [1] has indicated the existence of an uncoun- 
table collection of mutually exclusive tree-like continua in $E^{2}$ such that no one of them is chainable. By Theorem 9, for any uncountable collection $G$ of mutually exclusive homeomorphic continua in $E^{2}$, there exists a sequence of elements of $G$ converging homeomorphically to an element of $G$. This suggests the following question, which is left unanswered. If $M$ is a tree-like continuum in $E^{2}$ such that there exists a sequence of mutually exclusive continua in $E^{2}$ converging homeomorphically to $M$, does $M$ have uncountably many mutually exclusive homeomorphic images in $E^{2}$ ?

THEOREm 9. If $G$ is an uncountable collection of mutually exclusive homeomorphic continua in $E^{n}$, then there exists a sequence of elements of $G$ which converges homeomorphically to an element of $G$.

By using Borsuk's theorem that, under the metric $d(f, g)=$ $\max _{x \in M} \rho(f(x), g(x))$, the space of all continuous transformations of a compact metric space $M$ into a separable metric space is separable [5, Theorem 2], Theorem 9 can be proved by the method Bing [4] has indicated for the case where $G$ is a collection of arcs in $E^{2}$.

THEOREm 10. If $G$ is an uncountable collection of mutually exclusive tree-like continua in $E^{2}$, then all except a countable number of continua of $G$ have width zero.

Proof. It is sufficient to show that some continuum of $G$ has width zero. Suppose that no continuum of $G$ has width zero. It follows from Theorem 1 that there is a positive number $\delta$ and an uncountable subcollection $G^{\prime}$ of $G$ such that each continuum of $G^{\prime}$ has a width greater than $\delta$. But this is contrary to Theorem 4 since there is a convergent sequence of elements of $G^{\prime}$.

5. A remark on homogeneous decomposable plane continua. F. B. Jones [11] has shown that every nondegenerate homogeneous decomposable plane continuum has a continuous decomposition $G$ such that $G$ is a simple closed curve with respect to its elements and each element of $G$ is a homogeneous tree-like continuum. Jones' question as to whether each element of $G$ would be a pseudo-arc has not been answered, but Bing [3] has shown that this would be the case if each element of $G$ were linearly chainable. It follows from Theorem 10 that each element of $G$ has width zero. This suggests the following question. Is a homogeneous tree-like continuum chainable if it has width zero?

Added in proof. The author has recently shown that every homogeneous tree-like continuum in $E^{2}$ has width zero hereditarily and that a tree-like continuum has width zero hereditarily if and only if it is 
atriodic. These results will be presented in another paper.

\section{BIBLIOGRAPHY}

1. R. D. Anderson, Hereditarily indecomposable plane continua, Bull. Amer. Math. Soc., Abstract 57-3-236.

2. R. H. Bing, Snake-like continua, Duke Math. J., 18 (1951), 653-663.

3. - Each homogeneous nondegenerate chainable continuum is a pseudo-arc, Proc. Amer. Math. Soc., 10 (1959), 345-346.

4. - A simple closed curve is the only homogeneous bounded plane continuum that contains an arc, Canad. J. Math., 12 (1960), 209-230.

5. Karol Borsuk, Sur les rétractes, Fund. Math., 17 (1931), 152-170.

6. C. E. Burgess, Continua and their complementary domains in the plane, Duke Math. J., 18 (1951), 901-917.

7. Collections and sequences of continua in the plane, Pacific J. Math., 5 (1955), 325-333.

8. - Chainable continua and indecomposability, Pacific J. Math., 9 (1959), 653-659.

9. - Homogeneous continua which are almost chainable, Canad. J. Math., 13 (1961).

10. Samuel Eilenberg, Transformations continues en circonférence et la topologie du plan, Fund. Math., 26 (1936), 61-112.

11. F. B. Jones, On a certain type of homogeneous plane continuum, Proc. Amer. Math. Soc., 6 (1955), 735-740.

12. R. L. Moore, Concerning the common boundary of two domains, Fund. Math., 6 (1924), 203-213.

13. Concerning triods in the plane and the junction points of plane continua, Proc. Nat. Acad. Sci., 14 (1928), 85-88.

14. J. H. Roberts, Concerning atriodic continua, Monatsh. Math., 37 (1930), 223-230.

UNIVERSITY OF UTAH 


\title{
PACIFIC JOURNAL OF MATHEMATICS
}

\author{
EDITORS
}

\author{
Ralph S. Phillips \\ Stanford University \\ Stanford, California \\ F. H. Brownell \\ University of Washington \\ Seattle 5, Washington
}

\author{
A. L. Whiteman \\ University of Southern California \\ Los Angeles 7, California \\ L. J. PAige \\ University of California \\ Los Angeles 24, California
}

ASSOCIATE EDITORS
E. F. BECKENBACH
D. DERRY
H. L. ROYDEN
E. G. STRAUS
T. M. CHERRY
M. OHTSUKA
E. SPANIER
F. WOLF

\section{SUPPORTING INSTITUTIONS}

\author{
UNIVERSITY OF BRITISH COLUMBIA \\ CALIFORNIA INSTITUTE OF TECHNOLOGY \\ UNIVERSITY OF CALIFORNIA \\ MONTANA STATE UNIVERSITY \\ UNIVERSITY OF NEVADA \\ NEW MEXICO STATE UNIVERSITY \\ OREGON STATE COLLEGE \\ UNIVERSITY OF OREGON \\ OSAKA UNIVERSITY \\ UNIVERSITY OF SOUTHERN CALIFORNIA
}

\author{
STANFORD UNIVERSITY \\ UNIVERSITY OF TOKYO \\ UNIVERSITY OF UTAH \\ WASHINGTON STATE COLLEGE \\ UNIVERSITY OF WASHINGTON \\ * * * * \\ AMERICAN MATHEMATICAL SOCIETY \\ CALIFORNIA RESEARCH CORPORATION \\ HUGHES AIRCRAFT COMPANY \\ SPACE TECHNOLOGY LABORATORIES \\ NAVAL ORDNANCE TEST STATION
}

Mathematical papers intended for publication in the Pacific Journal of Mathematics should be typewritten (double spaced), and the author should keep a complete copy. Manuscripts may be sent to any one of the four editors. All other communications to the editors should be addressed to the managing editor, L. J. Paige at the University of California, Los Angeles 24, California.

50 reprints per author of each article are furnished free of charge; additional copies may be obtained at cost in multiples of 50 .

The Pacific Journal of Mathematics is published quarterly, in March, June, September, and December. The price per volume (4 numbers) is $\$ 12.00$; single issues, $\$ 3.50$. Back numbers are available. Special price to individual faculty members of supporting institutions and to individual members of the American Mathematical Society: $\$ 4.00$ per volume; single issues, $\$ 1.25$.

Subscriptions, orders for back numbers, and changes of address should be sent to Pacific Journal of Mathematics, 103 Highland Boulevard, Berkeley 8, California.

Printed at Kokusai Bunken Insatsusha (International Academic Printing Co., Ltd.), No. 6, 2-chome, Fujimi-cho, Chiyoda-ku, Tokyo, Japan.

\section{PUBLISHED BY PACIFIC JOURNAL OF MATHEMATICS, A NON-PROFIT CORPORATION}

The Supporting Institutions listed above contribute to the cost of publication of this Journal, but they are not owners or publishers and have no responsibility for its content or policies. 


\section{Pacific Journal of Mathematics}

\section{Vol. 11, No. 2 December, 1961}

Tsuyoshi Andô, Convergent sequences of finitely additive measures........

Richard Arens, The analytic-functional calculus in commutative topological algebras..........................................

Michel L. Balinski, On the graph structure of convex polyhedra in

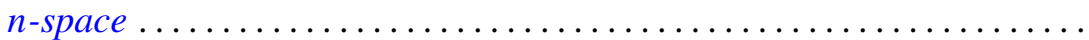

R. H. Bing, Tame Cantor sets in $E^{3}$...

Cecil Edmund Burgess, Collections and sequences of continua in the plane.

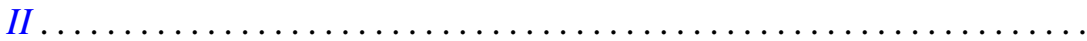

J. H. Case, Another 1-dimensional homogeneous continuum which contains an $\operatorname{arc}$

Lester Eli Dubins, On plane curves with curvature ................. 471

A. M. Duguid, Feasible flows and possible connections .............. 483

Lincoln Kearney Durst, Exceptional real Lucas sequences ................ 489

Gertrude I. Heller, On certain non-linear opeartors and partial differential equations........................................

Calvin Virgil Holmes, Automorphisms of monomial groups

Wu-Chung Hsiang and Wu-Yi Hsiang, Those abelian groups characterized by their completely decomposable subgroups of finite rank ..........

Bert Hubbard, Bounds for eigenvalues of the free and fixed membrane by finite difference methods .........................

D. H. Hyers, Transformations with bounded mth differences. .

Richard Eugene Isaac, Some generalizations of Doeblin's decomposition

John Rolfe Isbell, Uniform neighborhood retracts ..........

Jack Carl Kiefer, On large deviations of the empiric D. F. of vector chance variables and a law of the iterated logarithm...

Marvin Isadore Knopp, Construction of a class of modular functions and forms. II. . .

Gunter Lumer and R. S. Phillips, Dissipative operators in a Banach space....

Nathaniel F. G. Martin, Lebesgue density as a set function ...

Shu-Teh Chen Moy, Generalizations of Shannon-McMillan theorem ...

Lucien W. Neustadt, The moment problem and weak convergence in $L^{2}$

Kenneth Allen Ross, The structure of certain measure algebras...

James F. Smith and P. P. Saworotnow, On some classes of scalar-product algebras.

Dale E. Varberg, On equivalence of Gaussian measures. . 